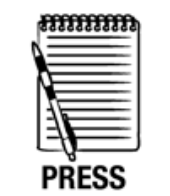

RELEASE

\title{
Colorectal cancer screening with odour material by canine scent detection
}

\author{
Hideto Sonoda, ${ }^{1,6}$ Shunji Kohnoe, ${ }^{1,6}$ Tetsuro Yamazato, ${ }^{2}$ Yuji Satoh, ${ }^{3}$ Gouki Morizono, \\ Kentaro Shikata, ${ }^{5}$ Makoto Morita, ${ }^{6}$ Akihiro Watanabe, ${ }^{6}$ Masaru Morita, ${ }^{1}$ \\ Yoshihiro Kakeji, ${ }^{1}$ Fumio Inoue, ${ }^{4}$ Yoshihiko Maehara ${ }^{1}$
}

\begin{abstract}
${ }^{1}$ Department of Surgery and Science, Kyushu University at Fukuoka, Fukuoka, Japan ${ }^{2}$ Department of Internal Medicine, Arita Kyoritsu Hospital at Arita, Saga, Japan ${ }^{3}$ St. Sugar Cancer Sniffing Dog Training Center at Minamibousou, Chiba, Japan ${ }^{4}$ Department of General Surgery, Arita Kyoritsu Hospital at Arita, Saga, Japan ${ }^{5}$ Department of Internal Medicine, Fukuoka Dental College Hospital at Fukuoka, Fukuoka, Japan

${ }^{6}$ Department of General Surgery, Fukuoka Dental College Hospital at Fukuoka, Fukuoka, Japan
\end{abstract}

\section{Correspondence to}

Dr Hideto Sonoda, Department of Surgery and Science Graduate School of Medicine Kyushu University 3-1-1 Midashi,Higashi-ku, Fukuoka 812-8582, Japan; hsonoda@ surg2.med.kyushu-u.ac.jp

Revised 23 November 2010 Accepted 7 December 2010 Published Online First 31 January 2011

\section{ABSTRACT}

Objective Early detection and early treatment are of vital importance to the successful treatment of various cancers. The development of a novel screening method that is as economical and non-invasive as the faecal occult blood test (FOBT) for early detection of colorectal cancer (CRC) is needed. A study was undertaken using canine scent detection to determine whether odour material can become an effective tool in CRC screening. Design Exhaled breath and watery stool samples were obtained from patients with CRC and from healthy controls prior to colonoscopy. Each test group consisted of one sample from a patient with CRC and four control samples from volunteers without cancer. These five samples were randomly and separately placed into five boxes. A Labrador retriever specially trained in scent detection of cancer and a handler cooperated in the tests. The dog first smelled a standard breath sample from a patient with CRC, then smelled each sample station and sat down in front of the station in which a cancer scent was detected.

Results 33 and 37 groups of breath and watery stool samples, respectively, were tested. Among patients with $\mathrm{CRC}$ and controls, the sensitivity of canine scent detection of breath samples compared with conventional diagnosis by colonoscopy was 0.91 and the specificity was 0.99 . The sensitivity of canine scent detection of stool samples was 0.97 and the specificity was 0.99 . The accuracy of canine scent detection was high even for early cancer. Canine scent detection was not confounded by current smoking, benign colorectal disease or inflammatory disease.

Conclusions This study shows that a specific cancer scent does indeed exist and that cancer-specific chemical compounds may be circulating throughout the body. These odour materials may become effective tools in CRC screening. In the future, studies designed to identify cancer-specific volatile organic compounds will be important for the development of new methods for early detection of CRC.

\section{INTRODUCTION}

Despite rapid progress in examination technology and therapy for colorectal cancer (CRC), this disease constitutes a significant proportion of the global burden of cancer morbidity and mortality. ${ }^{1}$ Multiple professional organisations have recommended screening for $\mathrm{CRC}^{1-5}$ and many previous studies have demonstrated the cost-effectiveness of CRC screening. ${ }^{6-11}$ Moreover, patients with familial adenomatous polyposis or Lynch syndrome are recommended to undergo frequent colonoscopy

\section{Significance of this study}

What is already known about this subject?

- Canine olfactory detection of cancer has been reported for melanoma as well as bladder, lung, breast and ovarian cancer.

- It is reported that dogs can be trained to distinguish breath samples of patients with lung and breast cancer from those of control volunteers and to distinguish ovarian cancer tissues from control tissues with high accuracy.

- Several volatile organic compounds have been identified as candidate substances for early detection of cancer using gas chromatography/ mass spectroscopy in the exhaled breath of patients with lung and breast cancer; while these data are promising, they are preliminary findings that should be interpreted with caution.

What are the new findings?

- In patients with colorectal cancer (CRC) and controls, the sensitivity of canine scent detection of breath samples compared with conventional diagnosis by colonoscopy was 0.91 and the specificity was 0.99 .

- The sensitivity of canine scent detection of watery stool samples was 0.97 and the specificity was 0.99 .

- The accuracy of canine scent detection was even higher for early-stage cancers.

- Canine scent detection was not confounded by current smoking, benign colorectal disease, inflammatory disease or the presence of human haemoglobin or transferrin.

\section{How might it impact on clinical practice in the foreseeable future?}

- Odour materials may become effective tools in CRC screening. In the future, studies designed to identify cancer-specific volatile organic compounds will be important for the development of new methods for early detection of CRC.

- Identification of cancer-specific volatile organic compounds will lead to solving the biological character of cancer.

to reduce the morbidity and mortality associated with CRC. ${ }^{12-15}$ Early detection and early treatment are critical for the successful treatment of cancer and are excellent means for reducing both
This paper is freely availabl gut.bmi.com/site/about/ unlocked.xhtml 
the economic burden and mortality. CRC therefore represents an excellent example of the value of early detection.

The faecal occult blood test (FOBT) is currently the most economic and non-invasive screening method for CRC, and many patients with positive results subsequently undergo total colonoscopy leading to a reduced incidence and mortality of CRC. ${ }^{23616}$ However, the positive predictive value of FOBT for CRC is approximately $10 \%,{ }^{17-2016} 21$ considering the impact of closer examination by colonoscopy or barium enema. The development of a novel more effective CRC screening method that is as economical and non-invasive as FOBT is therefore needed.

There have been several anecdotal reports of canine scent detection of skin cancer. ${ }^{22} 23$ Moreover, Willis et al reported that dogs can distinguish urine from patients with bladder cancer with a mean success rate of $41 \%,{ }^{24}$ while McCulloch et al reported that ordinary household dogs can be trained to distinguish breath samples of patients with lung and breast cancer from those of control volunteers with high accuracy (sensitivity and specificity of 0.99 and 0.99 in lung cancer and 0.88 and 0.98 in breast cancer, respectively). ${ }^{25}$ Horvath et al reported that, among ovarian cancer tissues and control tissues, the sensitivity was $100 \%$ and the specificity was $97.5 \%{ }^{26}$

In the present study we have confirmed the accuracy of canine scent detection of breath samples and evaluated canine scent detection of watery stool samples from patients with CRC. We also examined whether the diagnostic performance of dogs is affected by age, smoking, disease stage, cancer site, inflammation or bleeding in patients with cancer or control individuals.

\section{METHODS}

\section{Patient and control sample donors}

Patients were enrolled from 20 June 2008 to 20 May 2009 at the Fukuoka Dental College Medical and Dental Hospital and Arita Kyoritsu Hospital. To prepare for colonoscopy, subjects ingested 21 of a balanced electrolyte and polyethylene glycol 4000 solution (Niflec, Ajinomoto Pharma, Tokyo, Japan). Patients were required to be $>20$ years old. Patients and controls completed a questionnaire about factors that could influence volatile molecules in the breath or watery stool samples including age, physical symptoms (eg, abdominal pain or distention, bloody faeces, constipation, diarrhoea, body weight loss and abdominal tumour), history of cancer treatment, present use of anticoagulants and smoking within the previous 2 weeks. Colorectal diseases diagnosed primarily by colonoscopy were recorded in detail and pathological examination of biopsy samples was performed if necessary. Patients who had undergone cancer surgery within the previous year, those who did not undergo examination for cancer recurrence despite having undergone cancer surgery more than 5 years previously and those currently receiving chemotherapy were excluded. A serial number was written on each sample at the time of collection to identify individual information.

\section{Breath sampling}

Breath samples were acquired from the beginning to the end of exhalation. Each subject exhaled between 100 and $200 \mathrm{ml}$ into a breath sampling bag (Otsuka Pharmaceutical Company, Tokyo, Japan). The bags were then fitted with their end caps and sealed in ordinary grocery store Ziploc-style bags at $4^{\circ} \mathrm{C}$ until presentation to the dog. Only one sample was collected from each participant.

\section{Watery stool sampling}

A $50 \mathrm{ml}$ watery stool sample was obtained by suction during colonoscopy using a trachea aspirator kit (Sumitomo Bakelite
Company, Tokyo, Japan) between the rectum and descending colon and preserved at $-80^{\circ} \mathrm{C}$ until filtration. The sample was filtered with qualitative filter paper No. 2 (diameter $110 \mathrm{~mm}$; Tokyo Roshi Kaisha, Tokyo, Japan) and further filtered by Millex GP filter (pore size $0.22 \mu \mathrm{m}$, diameter $33 \mathrm{~mm}$, non-sterile type; Millipore Co, Corrigiwohill, Co. Cork, Ireland). Next, $1 \mathrm{ml}$ of filtrated watery stool samples was enclosed in a $5 \mathrm{ml}$ brown glass screw cap bottle, sealed in an ordinary grocery store small Ziploc-style bag and enclosed in a $50 \mathrm{ml}$ polypropylene screw cap tube and kept at $-20^{\circ} \mathrm{C}$ until presentation to the dog. Only one sample was collected from each participant.

\section{Dog and training}

A specially trained 8-year-old female black Labrador retriever from the St Sugar Cancer Sniffing Dog Training Center (Chiba, Japan) participated in the study. The dog was trained for water rescue beginning in 2003 and then began training as a cancer detection dog in 2005.

Each cancer detection training session was considered complete when the dog could correctly distinguish between breath samples from a cancer patient and four controls consecutively in dozens of trials. The fundamental training method was a reward-based approach in which the correct behaviour is rewarded by simultaneous play with a tennis ball. The breath samples used in the training steps beginning in 2005 were collected from several hundred patients with cancer and about 500 healthy volunteers recruited using the internet.

In the first training step in 2005, breath samples from one patient with oesophageal cancer and four controls were used. The samples were removed from the breath-sampling bag and placed into a paper cup which was then covered with a paper filter for 2 days. The five sample cups were placed on the floor by the handler. First, the dog smelled the standard breath sample from the patient with oesophageal cancer and then tried to identify the cup containing the oesophageal cancer scent from among the five sample cups. If the dog correctly identified the cancer sample it was rewarded with a tennis ball. A similar training session was conducted using breath samples from patients with lung cancer on the following day and was performed again using breath samples from patients with gastric cancer 2 days later.

In the second step the breath-sampling bag was used with the end cap on. Breath-sampling bags containing oesophageal, lung or gastric cancer scents originating from the same patient in the first step were chosen randomly for the 1-month training period.

In the third step the dog first smelled the breath sample from one type of cancer as a standard sample and then tried to identify the bag with the scent of another cancer. The training was then continued by adding samples of other types of cancers as well as controls.

The dog was already able to detect the following types of cancer in patient breath samples prior to initiation of the present study: oesophageal cancer, breast cancer, lung cancer, gastric cancer, pancreatic cancer, hepatocellular carcinoma, cholangiocarcinoma, colorectal cancer, prostate cancer, uterine cancer, ovarian cancer and bladder cancer.

\section{Training and test room}

The training and testing of the dog were conducted in a $4.8 \mathrm{~m} \times 8.0 \mathrm{~m}$ room with vinyl tiling and overhead fluorescent and natural window lighting. The room was not climate controlled and average ambient temperatures during the study (November 2008 through June 2009) ranged from $9^{\circ} \mathrm{C}$ to $20^{\circ} \mathrm{C}$. 
At the end of each study day the floor was wiped with water and a damp mop.

\section{Sample stations}

Five sample stations were positioned on the floor of the room in a single straight line spaced $52 \mathrm{~cm}$ apart. Each station consisted of a light blue painted wooden storage container measuring $27 \mathrm{~cm}$ wide, $30 \mathrm{~cm}$ long and $20 \mathrm{~cm}$ high. Each station also contained a wall $10 \mathrm{~cm}$ deep in which the breath sample bags or the watery stool sample bottles were placed. The top of each box was covered with metal netting to prevent the dog from directly contacting the samples.

\section{Breath and watery stool sample locations}

In a single trial, the dog passed and sniffed each of the five stations (one cancer sample and four control samples). Both patient and control samples were replaced with new samples after the dog had sniffed all five sample stations. Two to four trials were performed on each day of testing.

\section{Personnel}

The dog handler called the dog into the room and encouraged the dog to sniff the stations with the command 'Search!' after smelling the standard breath sample from the patient with CRC at each test. No other people were in the room.

\section{Classification of the dog's response}

Correct responses were (1) indication by sitting down in front of a sample station containing a cancer sample (a true positive in sensitivity calculations): and (2) sniffing but not indicating on a control sample (true negative). Incorrect responses were (1) indicating on a control sample (false positive); (2) sniffing but not indicating on a cancer sample (false negative); and (3) hesitation, considered to be an incomplete reaction to either cancer or control samples (either false positive or false negative depending on whether hesitation was on a cancer or control sample).

\section{Testing}

Tests were conducted from 13 November 2008 to 15 June 2009 because the dog's concentration tends to decrease during the hot summer season. For each test, completely new samples were used for both cases and controls; thus, each sample was only used a single time. For each trial we used a random number table to determine the location of the sample being tested in the lineup. Before the tests the sample was placed in either a new bag or new bottle to avoid contamination of the smell under preservation, and the number described in the sample was converted from the serial number into the test number at the same time. The laboratory assistants replaced the samples. The identity of the samples was blinded to the dog, the handler and the laboratory assistants, thereby making the study double-blind. One station contained a cancer sample and the remaining four stations contained control samples. At the tests the dog smelled the standard breath sample of CRC origin, after which the dog passed the side of some boxes and sat down in front of the station determined to contain a cancer scent, and then the handler noted the result on an answer card on which the test number was written. The card was transmitted to Fukuoka Dental College by fax. After submission the answer was explained to the handler and the dog as soon as possible because the correct behaviour was rewarded with a tennis ball. The cards were collected by mail. During the tests the dog did not experience any adverse events, injuries or illness.

\section{Occult blood test of watery stool samples}

The ordinary FOBT kit, Quick Chaser Occult Blood Test Kit (Mizuho Medy, Saga, Japan) was used for simultaneous detection of human haemoglobin and human transferrin in watery stool samples. Watery stool samples were mixed with phosphate buffered saline and reacted on the test plate. The test result was judged by a laboratory medical technologist and a physician. The result was defined as positive when human haemoglobin and/or transferrin was positive.

\section{Statistical analysis}

Associations between questionnaire variables before colonoscopy were tested by the Welch $\mathrm{t}$ test or $\chi^{2}$ test. All statistical differences of $p<0.05$ were deemed significant. CRC staging was based on Union Internationale Contre le Cancer (UICC) criteria.

During testing, experimenters monitored each trial and recorded their observations on a digital versatile disc. The entire dog performance dataset was audited for accuracy by comparing answer cards with digital versatile disc records.

Diagnostic accuracy was calculated as sensitivity and specificity of the dog's indication of samples compared with the true diagnosis confirmed by colonoscopy. Thus, the sensitivity (or the true positive rate) of the tests is the proportion of cancer samples correctly identified by the dog while the specificity (or the true negative rate) is the proportion of control samples negatively indicated by the dog.

\section{RESULTS}

Forty-eight samples were obtained from patients with CRC, 203 samples from volunteers with no history of cancer and 55 samples from patients with a previous history of cancer. For breath tests, 33 samples from patients with CRC and 132 samples from control volunteers were used and, for watery stool tests, 37 samples were used from patients with CRC and 148 samples from control volunteers. Each sample was only used once during testing. The background characteristics were well matched between cancer patients and control individuals, except for age (table 1). Approximately half of the controls ( $47 \%$ of breath samples, $50 \%$ of watery stool samples) had colorectal polyps and a small proportion of the controls $(6.1 \%$ of breath samples, $10.5 \%$ of watery stool samples) had bleeding or inflammatory colorectal disease, including ischaemic colitis, nonpeculiar colitis or ulcer, ulcerative colitis, diverticular bleeding, mesenteric panniculitis and chronic appendicitis (table 2).

\section{Diagnostic accuracy: sensitivity and specificity in testing}

To calculate sensitivity and specificity we designated the dog's response to each sample sniffed as the unit of analysis (defined above). Comparison of the judgement of the canine scent in the breath or watery stool samples with a colonoscopy-confirmed conventional diagnosis among cancer patients and controls gave an overall sensitivity for canine scent detection of 0.91 and 0.97 , respectively, while the overall specificity was 0.99 and 0.99 , respectively (table 3 ).

During the course of 74 tests, four discrepancies occurred between colonoscopy and the canine scent detection results (1/38 watery stool tests and $3 / 36$ breath tests). The discrepancies occurred in the 22nd, 26th, 33rd and 45th tests. Moreover, these discrepancies occurred in any of four tests on the aforementioned test days. Interestingly, the watery stool sample that yielded a discrepancy came from an individual whose breath sample also resulted in a discrepancy. Samples from the three patients who did not have colon cancer but whose samples yielded positive results in the watery stool and/or breath tests 
Table 1 Background characteristics of participants

\begin{tabular}{|c|c|c|c|c|c|c|}
\hline & \multicolumn{3}{|c|}{ Breath samples } & \multicolumn{3}{|c|}{ Watery stool samples } \\
\hline & $\begin{array}{l}\text { Cancer } \\
(n=33)\end{array}$ & $\begin{array}{l}\text { Control } \\
(n=132)\end{array}$ & p Value & $\begin{array}{l}\text { Cancer } \\
(\mathrm{n}=37)\end{array}$ & $\begin{array}{l}\text { Control } \\
(n=148)\end{array}$ & p Value \\
\hline Gender (male) & 15 & 64 & 0.75 & 16 & 86 & 0.10 \\
\hline Age (years) & $71.0(53-95)$ & $65.0(25-91)$ & 0.001 & $70.2(41-95)$ & $64.6(25-91)$ & 0.035 \\
\hline \multicolumn{7}{|l|}{ Complaint before colonoscopy } \\
\hline Abdominal pain or distention & 6 & 20 & 0.67 & 10 & 22 & 0.08 \\
\hline Bloody faeces & 5 & 19 & 0.91 & 6 & 19 & 0.59 \\
\hline Constipation & 6 & 24 & 1.0 & 7 & 24 & 0.69 \\
\hline Diarrhoea & 3 & 8 & 0.53 & 4 & 14 & 0.95 \\
\hline Body weight loss & 2 & 8 & 0.68 & 3 & 4 & 0.29 \\
\hline Abdominal tumour & 2 & 1 & 0.19 & 2 & 1 & 0.19 \\
\hline No complaint & 4 & 21 & 0.59 & 3 & 28 & 0.18 \\
\hline Past history of colorectal disease & 1 & 20 & 0.11 & 2 & 22 & 0.13 \\
\hline Anticoagulant therapy & 1 & 14 & 0.31 & 1 & 18 & 0.16 \\
\hline Smoked within 2 weeks & 3 & 28 & 0.18 & 4 & 32 & 0.21 \\
\hline
\end{tabular}

did not result in discrepancies on the other tests. These three patients showed no signs of cancer during the 24 months following sampling.

\section{Comparison between FOBT results and the dog's judgement}

The results of watery stool tests and conventional FOBTs were compared to determine whether canine scent judgement relied on blood. No correlation between canine scent judgement and FOBT results was observed in patients with cancer $(p=0.51)$ or controls $(p=0.68$, table 4$)$. The sensitivity and specificity of FOBT with respect to CRC detection was 0.70 and 0.85 , respectively.

\section{Mixture test of watery stool samples}

To determine whether a specific cancer scent exists or if a particular natural scent vanishes in patients with cancer, we combined five watery stool samples into single mixture samples. One of the mixture samples was composed of one sample of cancer origin and four control samples, while four mixture samples consisted of five control samples each. Tests were performed three times as described above. Cancer types included stage IIIa ascending colon cancer, stage II transverse colon cancer and stage 0 rectal cancer. The results of all three tests were correct.

\section{DISCUSSION}

We used the excellent ability of dogs to distinguish between different scents to examine whether odour materials can be used in the diagnosis of CRC. This study represents the first step

Table 2 Disease in control cases

\begin{tabular}{lccccc}
\hline & \multicolumn{2}{l}{ Breath samples } & & \multicolumn{2}{c}{ Watery stool samples } \\
\cline { 2 - 3 } & Number & Rate & & Number & Rate \\
\hline Polyps & 62 & $47.0 \%$ & & 74 & $50.0 \%$ \\
Diverticula & 24 & $18.2 \%$ & & 44 & $29.7 \%$ \\
Haemorrhoids & 4 & $3.0 \%$ & & 7 & $4.7 \%$ \\
Ischaemic colitis & 3 & $2.3 \%$ & & 4 & $2.7 \%$ \\
Non-peculiar colitis or ulcers & 2 & $1.5 \%$ & & 4 & $2.7 \%$ \\
Ulcerative colitis & 2 & $1.5 \%$ & & 4 & $2.7 \%$ \\
Diverticular bleeding & 0 & $0 \%$ & & 1 & $0.6 \%$ \\
Mesenteric panniculitis & 1 & $0.8 \%$ & & 1 & $0.6 \%$ \\
Infectious colitis & 0 & $0 \%$ & & 1 & $0.6 \%$ \\
Chronic appendicitis & 0 & $0 \%$ & & 1 & $0.6 \%$ \\
Lynch syndrome & 1 & $0.8 \%$ & & 1 & $0.6 \%$ \\
\hline
\end{tabular}

Multiple answers were allowed for benign disease. towards the development of an early detection system using odour materials from patients with CRC.

McCulloch et al previously reported the use of canine scent judgement of breath samples for distinguishing between controls and patients with lung and breast cancer. ${ }^{25}$ We therefore evaluated canine scent detection of CRC first with breath samples and then with watery stool samples, which has not been previously reported. We hypothesised that the watery stool sample scent might be strong because the samples were collected from nearer to the cancer than were the breath samples. Conversely, the watery stool sample scents might have been masked by various other scents such as short-chain fatty acids and sulfides present in the stool. Moreover, we were able to compare scent detection with the FOBT by using the same stool sample and to inspect it more quantitatively than using breath samples. Indeed, despite various disrupting scents, the watery stool sample tests were superior to the breath sample tests.

We compared the accuracy of canine scent judgement with colonoscopic diagnosis using breath samples and watery stool samples from patients with CRC and controls. For breath tests, the sensitivity and specificity was 0.91 and 0.99 , respectively and, for watery stool tests, the sensitivity and specificity was 0.97 and 0.99 , respectively. Moreover, canine scent judgement even appeared to be highly accurate for early-stage CRC.

Canine scent judgement was not confounded by current smoking, benign colorectal polyps, inflammation or infection. A statistically significant difference in age distribution was noted between cancer patients and controls ( $p=0.001$ for breath tests, $p<0.05$ for watery stool tests). We therefore reanalysed samples from patients and controls aged $<80$ years. In this subset analysis, no correlation between age and cancer status was observed ( $p=0.09$ for 19 breath tests, $p=0.93$ for 24 watery stool tests). The sensitivity and specificity in this subset analysis for breath tests was 0.95 and 0.99 , respectively, while the sensitivity and specificity were both 1.00 for watery stool tests.

There were four discrepancies among the 74 tests including one of 38 watery stool tests and three of 36 breath tests between the colonoscopy and canine cancer detection results. It is difficult to specify the cause of these discrepancies owing to their limited number. The discrepancies occurred in the 22nd, 26th, $33 r d$ and 45 th tests, not necessarily in the early tests. Moreover, these discrepancies occurred in any of the four tests on the aforementioned test days, not necessarily in those taken late in the day. No correlation between the discrepancies and training effect or fatigue was suspected. Interestingly, the watery stool 
Table 3 Accuracy of canine scent detection by site and stage of cancer

\begin{tabular}{|c|c|c|c|c|c|c|c|c|c|c|c|c|}
\hline \multirow[b]{2}{*}{ Site/stage } & \multicolumn{6}{|c|}{ Breath samples } & \multicolumn{6}{|c|}{ Watery stool samples } \\
\hline & 0 & 1 & II & III & IV & Total & 0 & I & II & III & IV & Total \\
\hline Appendix & & & $1 / 1$ & & & $1 / 1$ & & & $1 / 1$ & & & $1 / 1$ \\
\hline Ascending & $1 / 1$ & & $3 / 3$ & $4 / 5^{*}$ & $2 / 2$ & $10 / 11^{*}$ & $1 / 1$ & $1 / 1$ & $3 / 3$ & $5 / 5$ & $2 / 2$ & $12 / 12$ \\
\hline Transverse & $1 / 1$ & & & & & $1 / 1$ & $1 / 1$ & & $1 / 1$ & & & $2 / 2$ \\
\hline Descending & & & $1 / 1$ & $1 / 1$ & & $2 / 2$ & & & $1 / 1$ & $1 / 1$ & & $2 / 2$ \\
\hline $\mathrm{R}+\mathrm{S}$ & & & & $1 / 1$ & & $1 / 1$ & & & & $1 / 1$ & & $1 / 1$ \\
\hline Total & $4 / 4$ & $6 / 8$ & $7 / 7$ & $9 / 10^{*}$ & $7 / 7$ & $33 / 36^{*}$ & $6 / 6$ & $5 / 6$ & $9 / 9$ & $11 / 11$ & $6 / 6$ & $37 / 38$ \\
\hline Sensitivity & 1 & 0.67 & 1 & 0.89 & 1 & 0.91 & 1 & 0.8 & 1 & 1 & 1 & 0.97 \\
\hline Specificity & 1 & 0.92 & 1 & 1 & 1 & 0.99 & 1 & 0.95 & 1 & 1 & 1 & 0.99 \\
\hline
\end{tabular}

Number of true positives/total test number are shown.

*Hesitation occurred.

$\mathrm{R}+\mathrm{S}$, double rectal and sigmoid colon cancer.

sample that yielded a discrepancy came from an individual whose breath sample also resulted in a discrepancy. Moreover, when the watery stool test was repeated with different controls, the dog hesitated a few times and then barely detected the rectal cancer sample, leading to negative results in both the breath and watery stool tests. It is thought that some cancers are difficult to detect by canine scent.

No correlation between canine scent judgement and human haemoglobin and transferrin was observed. Thus, canine scent judgement does not appear to depend on human haemoglobin or transferrin scents. All canine scent judgements of watery stool sample mixture tests were correct, indicating that a specific cancer scent indeed exists. The volatile organic compounds (VOCs) detected during canine scent judgement presumably occur early in the pathogenesis of CRC. As canine scent judgement can be used on both breath samples and watery stool samples, these chemical compounds may be circulating throughout the body.

To determine whether a specific cancer scent exists or if a particular natural scent disappears in patients with cancer, mixtures that included watery stool samples were tested. Tests were performed three times as described above. The results of all three tests were correct, thereby suggesting that a specific cancer scent indeed exists.

Canine scent judgement for distinguishing between cancer patients and controls has been reported for other cancer types. ${ }^{24-26}$ We obtained breath and watery stool samples from patients with breast, stomach and prostate cancer who also underwent colonoscopy. We also tested these samples using breath samples from patients with CRC as the standard. Canine scent judgement yielded correct answers for these cancers as well (data not shown), suggesting that common scents may exist among various cancer types. This is consistent with the results of McCulloch et al which demonstrated the utility of canine scent detection for lung and breast cancer $^{25}$; however, these results are not inconsistent with the existence of cancerspecific scents.

Table 4 Relationship between canine scent detection and human haemoglobin and transferrin

\begin{tabular}{lllcl}
\hline \multirow{2}{*}{$\begin{array}{l}\text { Pathological } \\
\text { judgement }\end{array}$} & $\begin{array}{l}\text { Judgement } \\
\text { by dog }\end{array}$ & \multicolumn{2}{l}{$\begin{array}{l}\text { Judgement by faecal } \\
\text { occult blood test }\end{array}$} & \\
\cline { 2 - 4 } Cancer & Positive & Negative & p Value \\
& Positive & 25 & 11 & 0.51 \\
Control & Negative & 1 & 0 & \\
& Positive & 0 & 1 & 0.68 \\
& Negative & 22 & 125 & \\
\hline
\end{tabular}

It may be difficult to introduce canine scent judgement into clinical practice owing to the expense and time required for the dog trainer and for dog education. Scent ability and concentration vary between different dogs and also within the same dog on different days. Moreover, each dog can only conduct tests for a maximum of 10 years. It is therefore necessary to identify the cancer-specific VOCs detected by dogs and to develop an early cancer detection sensor that can be substituted for canine scent judgement.

Breath VOCs have been reported as biomarkers of other gastrointestinal disorders such as inflammatory bowel disease, ulcerative colitis and necrotising enterocolitis. ${ }^{27-30}$ However, these tests have not been widely accepted, largely due to technical difficulties in measuring the pressure of exhaled VOCs, the large variations in food intake, the variability in the required time for bacterial colonisation and the poor predictive value of a positive test.

Several VOCs have been identified as candidate substances for early detection of cancer using gas chromatography/mass spectroscopy (GC/MS) in the exhaled breath of patients with lung and breast cancer ${ }^{31-38}$; while these data are promising, they are preliminary findings that should be interpreted with caution. We intend to evaluate these VOCs by chemical analysis and with cancer detection dogs in the near future. Identification of cancerspecific VOCs will lead to solving the biological character of cancer. We hope that the results of the present study will provide encouragement for the development of cancer detection and solving the biological character of cancer using odour material.

Acknowledgements The authors thank Tomoe Fujino and Mika Ohnishi for their valuable technical assistance. The sponsor of the trial had no role in the design or conduct of the study, or in the analysis of the data. The corresponding author had full access to the data and had final responsibility for the decision to submit the data for publication. No compensation was given to subjects for providing breath samples or watery stool samples.

Funding This study was funded by Fukuoka Dental College.

Competing interests None.

Ethics approval This study was conducted with the approval of the institutional review boards at Fukuoka Dental College and Arita Kyoritsu Hospital and all subjects provided written informed consent.

Provenance and peer review Not commissioned; externally peer reviewed.

\section{REFERENCES}

1. Boyle PLB, ed. World Cancer Report 2008. Lyon, France: IARC Press, 2008.

2. US Preventive Services Task Force. Screening for colorectal cancer: U.S. Preventive Services Task Force recommendation statement. Ann Intern Med 2008;149:627-37. 
3. Winawer SJ, Zauber AG, Fletcher $\mathrm{RH}$, et al. Guidelines for colonoscopy surveillance after polypectomy: a consensus update by the US Multi-Society Task Force on Colorectal Cancer and the American Cancer Society. Gastroenterology 2006;130:1872-85.

4. US Preventive Services Task Force. Screening for colorectal cancer: recommendation and rationale. Ann Intern Med 2002:137:129-31.

5. Rex DK, Johnson DA, Lieberman DA, et al. Colorectal cancer prevention 2000: screening recommendations of the American College of Gastroenterology. American College of Gastroenterology. Am J Gastroenterol 2000;95:868-77.

6. Walsh JM, Terdiman JP. Colorectal cancer screening: clinical applications. JAMA 2003;289:1297-302

7. Frazier AL, Colditz GA, Fuchs CS, et al. Cost-effectiveness of screening for colorectal cancer in the general population. JAMA 2000;284:1954-61.

8. Sonnenberg A, Delco F, Inadomi JM. Cost-effectiveness of colonoscopy in screening for colorectal cancer. Ann Intern Med 2000:133:573-84.

9. Park SM, Yun YH, Kwon S. Feasible economic strategies to improve screening compliance for colorectal cancer in Korea. World J Gastroenterol 2005;11:1587-93

10. Shimbo T, Glick HA, Eisenberg JM. Cost-effectiveness analysis of strategies for colorectal cancer screening in Japan. Int J Technol Assess Health Care 1994; 10:359-75.

11. Wagner JL, Herdman RC, Wadhwa S. Cost effectiveness of colorectal cancer screening in the elderly. Ann Intern Med 1991;115:807-17.

12. Lynch HT, Lynch JF, Attard TA. Diagnosis and management of hereditary colorectal cancer syndromes: Lynch syndrome as a model. CMAJ 2009:181:273-80.

13. Kievit W, de Bruin JH, Adang EM, et al. Cost effectiveness of a new strategy to identify HNPCC patients. Gut 2005;54:97-102.

14. Lynch HT, de la Chapelle A. Hereditary colorectal cancer. N Engl J Med 2003;348:919-32.

15. Ramsey SD. Screening for the Lynch syndrome. N Engl J Med 2005;353:524-5; author reply 525

16. Mandel JS, Church TR, Bond JH, et al. The effect of fecal occult-blood screening on the incidence of colorectal cancer. N Engl J Med 2000;343:1603-7.

17. UK Colorectal Cancer Screening Pilot Group. Results of the first round of a demonstration pilot of screening for colorectal cancer in the United Kingdom. BMJ 2004;329:133

18. Hamilton W, Round A, Sharp D, et al. Clinical features of colorectal cancer before diagnosis: a population-based case-control study. Br J Cancer 2005:93:399-405

19. Manfredi S, Piette C, Durand G, et al. Colonoscopy results of a French regional FOBT-based colorectal cancer screening program with high compliance. Endoscopy 2008;40:422-7
20. Steele RJ, McClements PL, Libby G, et al. Results from the first three rounds of the Scottish demonstration pilot of FOBT screening for colorectal cancer. Gut 2009:58:530-5.

21. Ahlquist DA, Wieand HS, Moertel CG, et al. Accuracy of fecal occult blood screening for colorectal neoplasia. A prospective study using Hemoccult and HemoQuant tests. JAMA 1993;269:1262-7.

22. Williams H, Pembroke A. Sniffer dogs in the melanoma clinic? Lancet 1989;1:734

23. Church J, Williams H. Another sniffer dog for the clinic? Lancet 2001;358:930

24. Willis CM, Church SM, Guest CM, et al. Olfactory detection of human bladder cancer by dogs: proof of principle study. BMJ 2004;329:712.

25. McCulloch M, Jezierski T, Broffman M, et al. Diagnostic accuracy of canine scent detection in early- and late-stage lung and breast cancers. Integr Cancer Ther 2006;5:30-9.

26. Horvath G, Jarverud GA, Jarverud $\mathrm{S}$, et al. Human ovarian carcinomas detected by specific odor. Integr Cancer Ther 2008:7:76-80.

27. Cheu HW, Brown DR, Rowe MI. Breath hydrogen excretion as a screening test for the early diagnosis of necrotizing enterocolitis. Am J Dis Child 1989:143:156-9.

28. Pelli MA, Trovarelli G, Capodicasa $\mathrm{E}$, et al. Breath alkanes determination in ulcerative colitis and Crohn's disease. Dis Colon Rectum 1999;42:71-6.

29. Tibble JA, Sigthorsson G, Foster $\mathrm{R}$, et al. Use of surrogate markers of inflammation and Rome criteria to distinguish organic from nonorganic intestinal disease. Gastroenterology 2002;123:450-60.

30. Pelton NS, Tivey DR, Howarth GS, et al. A novel breath test for the non-invasive assessment of small intestinal mucosal injury following methotrexate administration in the rat. Scand J Gastroenterol 2004;9:1015-16.

31. O'Neill HJ, Gordon SM, O'Neill MH, et al. A computerized classification technique for screening for the presence of breath biomarkers in lung cancer. Clin Chem 1988;34:1613-18.

32. Phillips $\mathbf{M}$, Cataneo RN, Ditkoff BA, et al. Prediction of breast cancer using volatile biomarkers in the breath. Breast Cancer Res Treat 2006;99:19-21.

33. Preti G, Labows JN, Kostelc JG, et al. Analysis of lung air from patients with bronchogenic carcinoma and controls using gas chromatography-mass spectrometry. J Chromatogr 1988:432:1-11.

34. Phillips M, Cataneo RN, Ditkoff BA, et al. Volatile markers of breast cancer in the breath. Breast J 2003;9:184-91.

35. Gordon SM, Szidon JP, Krotoszynski BK, et al. Volatile organic compounds in exhaled air from patients with lung cancer. Clin Chem 1985:31:1278-82.

36. Phillips M, Greenberg J. Method for the collection and analysis of volatile compounds in the breath. J Chromatogr 1991;564:242-9.

37. Phillips M. Breath tests in medicine. Sci Am 1992;267:74-9

38. Phillips M, Sabas M, Greenberg J. Increased pentane and carbon disulfide in the breath of patients with schizophrenia. J Clin Pathol 1993:46:861-4. 